\title{
Nonlinear Modal Analysis of Frictional Ring Damper for Compressor Blisk
}

\author{
Yekai Sun,; \\ Department of Mechanical Engineering \\ Imperial College London \\ SW7 2AZ London, UK \\ Email: ys5113@imperial.ac.uk \\ Jie Yuan, Enora Denimal, Loïc Salles \\ Department of Mechanical Engineering \\ Imperial College London \\ SW7 2AZ London, UK
}

\begin{abstract}
The use of integrally blisk is becoming popular because of the advantages in aerodynamic efficiency and mass reduction. However, in an integrally blisk, the lack of the contact interface leads to a low structural damping compared to an assembled bladed-disk. One emerging damping technique for the integrally blisk is based on the use of friction ring damper which exploits the contact interfaces at the underneath of the disk. In this paper, three different geometries of the ring dampers are investigated for damping enhancement of a blisk. A full-scale compressor blisk is considered as a case study where a node to node contact model is used to compute the contact forces. The dynamic behaviour of the blisk with the ring damper is investigated by using nonlinear modal analysis which allows a direct estimation of the damping generated by the friction interface. The damping performance for the different ring dampers are evaluated and compared. It appears that the damping efficiency as well as the shift in the resonant frequency for the different geometries are highly related to the nodal diameter and contact pressure/gap distributed within contact interface. The geometry of the ring damper has significant impact on the damping performance.
\end{abstract}

\section{Introduction}

Gas turbine engine in aerospace industry becomes lighter and more efficient. Especially, the bladed disks within the gas turbine engine are almost driven to their structural limit due to the higher efficiency required. These bladed disks usually work under high vibrational stress, thermal stress and centrifugal stress. The high cycle fatigue caused by large vibrational stress is regarded as a major reason for most of the aero-engine failures [1]. However, the material damping and aerodynamic damping for bladed disk is

\footnotetext{
*Address all correspondence to this author.
}

relatively low and external damping is required to reduce the level of the vibration through the resonance. Frictional damping is a common external damping source within the turbomachinery bladed disk. Various frictional dampers have been investigated and designed in both numerical analysis and experimental works [2-5]. The kinematic description of the contact model for computation of friction has been wellestablished in literature [6].

The use of integrally blisk is very popular instead of the traditional bladed disks. The disk and blades are, in integrally blisk, manufactured in a single piece component leading to reduction of mass and a better aerodynamic performance. But the absence of contact interfaces gives a low internal damping. Therefore, an alternative damping source is introduced for the integrally blisk to add external friction interfaces for extra damping. It often consists in the addition of a frictional ring damper. The latter is located in the groove of the integrally blisk and is held against the disk by centrifugal forces. Similar frictional ring dampers are known as efficient dampers in other rotating structures such as labyrinth seals [7] and gears [8,9].

The dynamic analysis of the frictional ring damper for integrally blisk has been exploited by many researchers. A lumped parameter model was studied and the qualitative results were obtained in forced response analysis [10] and using nonlinear modes [11]. The interested readers can find some numerical analysis for the ring dampers using a Finite Element (FE) model in [12-15]. In addition, the comparison between the numerical simulation and experimental results were completed by Laxalde et al. [16]. The geometries of the frictional ring damper are seldom studied. In a recent published paper, a geometric optimisation of a V-shaped ring damper was investigated for forced responses in [17]. However, no studies are considering other geometries for the ring dampers. In this work, different geometries of the 


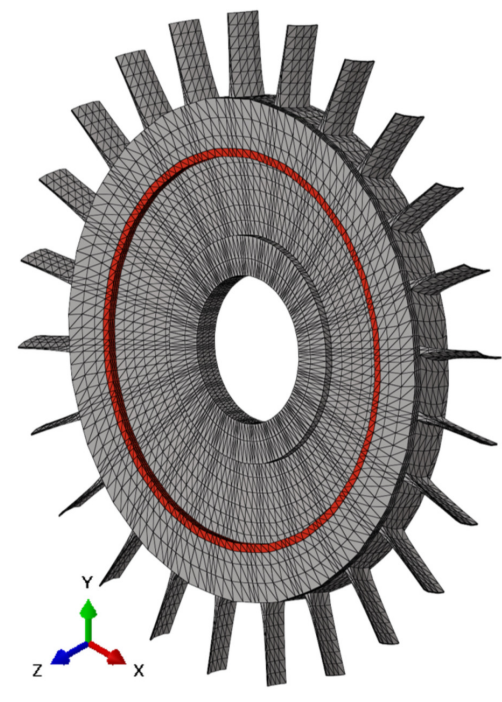

(a) Full Annulus

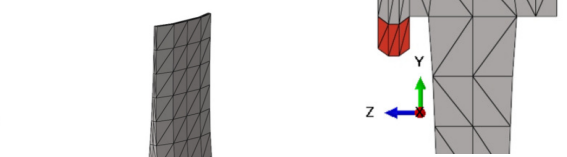

(a) Ring A

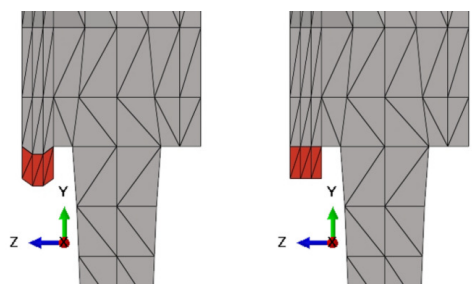

(b) Ring B

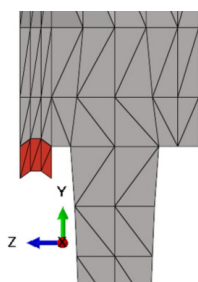

(c) Ring C
Fig. 2: View of the geometries of three ring dampers studied

tion [2, 25]. In addition, the continuation method is widely used to track the evolution of the system for a specific chasing parameter [26]. Hence, HBM integrated with continuation method is the numerical methodology used in this work to compute the steady state solution for nonlinear dynamic system.

The present work aims to study the influence of the ge-

Fig. 1: FE model of the system: (a) Integrally blisk model with ring damper (red); (b) A sector of blisk and ring damper (red) with cyclic symmetric boundaries (blue)

frictional ring damper are studied through Nonlinear Modal Analysis (NMA) and damping performances are quantified and evaluated. The present study can be considered as the pioneering work to explore the different geometries (shape of cross section) of the ring damper in dynamics regime with the consideration of contact friction.

The dynamic behaviour of systems with contact friction exhibits strong nonlinearity. NMA is known as an efficient methodology to study systems with nonlinearities, such as contact friction. Nonlinear modes have been defined by several researchers using different concepts $[18,19]$. The nonlinear modes in nonlinear damped system are named as damped Nonlinear Normal Mode (dNNM) [20], which are considered as the free vibration solutions for a dissipative autonomous system. Laxalde et al. [21] developed a numerical method using the concept of complex nonlinear mode for computation of dNNM. An energy dependency is introduced into the definition of the dNNM [21]. The dNNMs and other modal properties vary with the level of the energy of the mode. Further, a numerical method to compute a periodic based dNNMs, namely Extension of Periodic Motion Concept (EPMC), is developed by Krack [22]. The EPMC is used in the numerical computation for NMA. The dNNMs and other modal properties (resonant frequency and modal damping ratio) can be directly computed through NMA for a nonlinear system.

It is a complicated task to compute steady state solution for a nonlinear system. Therefore, various numerical methods were developed in literature in frequency domain [23]. The Harmonic Balance Method (HBM) with Alternating Frequency/Time (AFT) method is a classic frequency method for dynamic analysis of the nonlinear system [24]. It has been well implemented in nonlinear vibration problem for cyclic reduced blisk sector with contact fricometry of a frictional ring damper on the dynamic behaviour of an integrally blisk. The study is performed on a 3D FE model to represent the blisk and the ring damper, and three different geometries of the ring damper are considered. The resonance cases are identified through linear modal analysis and rotor dynamic analysis. The dNNMs, resonant frequency and modal damping ratio are computed through the NMA in a nonlinear vibration solver and used for the evaluation of the damping performance. This paper is organised as follows: the FE model is described in Section 2; then, the methodology for pre-processing is introduced in Section 3; after that, the numerical formulations for nonlinear vibration solver are briefly explained. Finally, the modal properties are investigated and the damping abilities are assessed and compared among different ring dampers.

\section{Finite Element Model}

The model used in this work is a three dimensional FE model. This blisk model is designed as a test case for the dynamic analysis of the frictional ring damper. This FE model of the blisk and ring damper is built using the commercial FE code ABAQUS and is represented in Fig.1, where the blisk is in grey and the ring damper is in red. The blisk and the ring damper are entirely made of titanium considered as a homogenous and isotropic material. A sector of the blisk and ring are shown in Fig.1(b) and there are 24 sectors in total. This model contains 1956 elements, where 1848 for blisk sector and 108 for the sector of ring damper. Each node has three translational Degree of Freedoms (DoFs). Three different geometries of ring dampers are considered and represented in Fig.2. One of them (Ring B) has a flat contact surface, and the others two are curved (see Fig.2(a, c)). To perform a dynamic analysis, the following assumptions are done:

1. Cyclic Symmetry: The blisk is assumed as a perfectly tuned structure which exhibits cyclic symmetry for the full annulus. A split ring damper is usually considered 


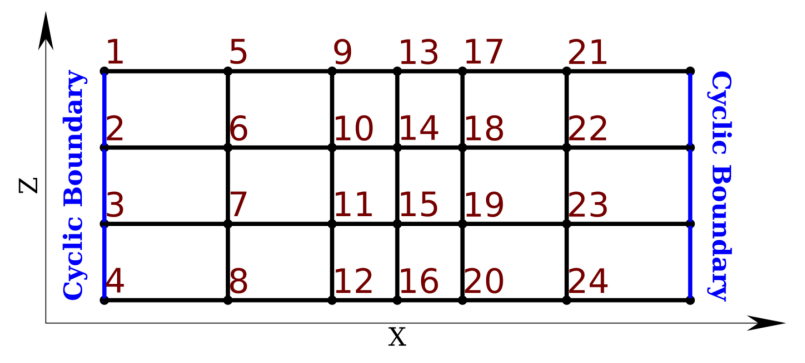

Fig. 3: X-Z plane of contact interface and node number

for the actual design and leads to a mistuning effect ${ }^{1}$. The ring damper has been proved to be efficient for the mistuned case [10], therefore, this mituning effect is ignored and the ring damper is assumed to be continuous and cyclic symmetric. The cross section of the ring is uniform along the full annulus.

2. Kinematic Nonlinearity: The integrally blisks are rigid enough to avoid kinematic nonlinearity at large vibration amplitude. Hence, kinematic nonlinearity is ignored in the nonlinear vibration analysis.

3. Different Ring Geometries: The groove of the blisk is different due to the different shapes of the rings as shown in Fig.2. For different ring dampers, the rotational radius and mass of the ring damper are kept as constant values for all geometries.

\section{Methodology for Pre-processing}

The FE model has been described in the previous section. In this section, the methodology used for preprocessing is explained in details. The flow chart of the pre-processing is shown in Fig.4. The objective of the preprocessing is to identify the resonance cases for nonlinear vibration analysis. A commercial FE solver ABAQUS is used for the following work. At the first place, a linear modal analysis is used for the FE blisk model (see Fig.1) to obtain the Nodal Diameter ${ }^{2}$ (ND) diagram. Further more, a classic Campbell diagram is obtained through a rotor dynamics analysis to take the centrifugal stiffening effect into consideration. Then, several resonance cases are identified through the Campbell diagram. After that, the blisk with different ring dampers are analysed with contact model under the resonance case and contact pressure/gap distribution within contact interface are obtained in nonlinear static analysis.

\subsection{Nodal diameter diagram}

A classic ND diagram is shown in Fig.5, which indicates the six natural frequencies $\omega_{n}$ against ND for the FE blisk model. The free vibrations of the blisk are indicated in the figure, which includes $1^{\text {st }}$ bending mode (1B), $2^{\text {nd }}$ bending mode (2B), $1^{\text {st }}$ torsion mode (1T) and first three families of

\footnotetext{
${ }^{1}$ Mistuning refers the break of symmetry for a cyclic symmetric system due to the small variation in their properties.

${ }^{2}$ Nodal diameter is defined as the number of diameters in a cyclic symmetric structure that have zero displacements.
}

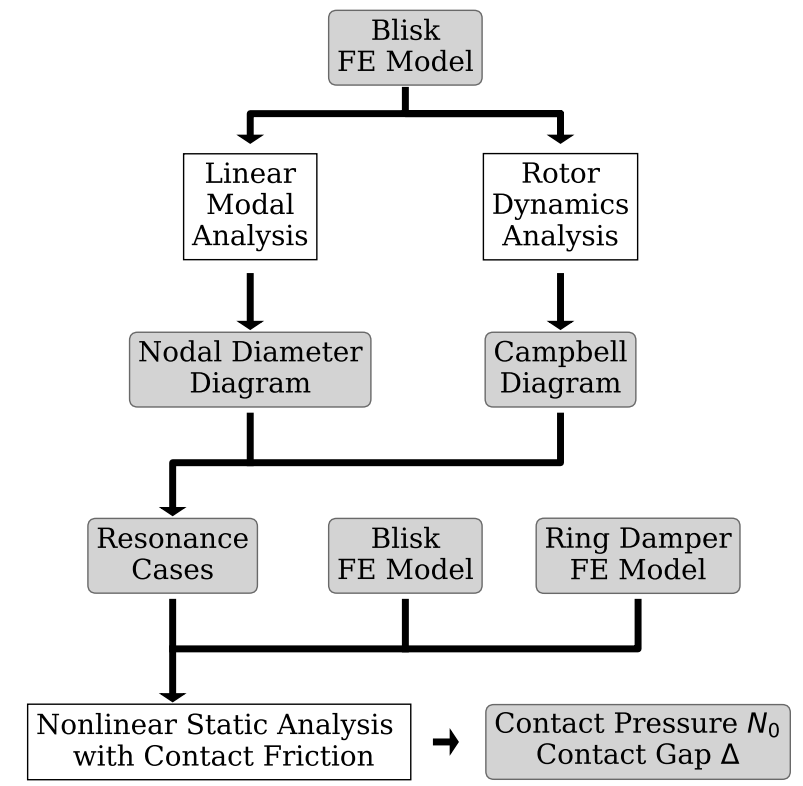

Fig. 4: Flow chart for pre-processing

mode dominated by disk part (1D, 2D, 3D). The modes shape of these four modes (ND 2 for 1B, 1D, 2B, 1T) are shown in Fig.6. In ND diagram, the natural frequencies $\omega_{n}$ of the mode family dominated by blade motion do not significantly vary with the ND, e.g. $1 \mathrm{~B}, 2 \mathrm{~B}$ and $1 \mathrm{~T}$. Whereas if the natural frequencies $\omega_{n}$ of the mode family changes dramatically with $\mathrm{ND}$, then the mode family is dominated by disk motion (e.g. 1D). The frictional damper is designed to be placed underneath the disk. Since the large displacements at the contact interface are expected, therefore the 1D modes (coloured in red) are of the most interest for the analysis of frictional ring damper.

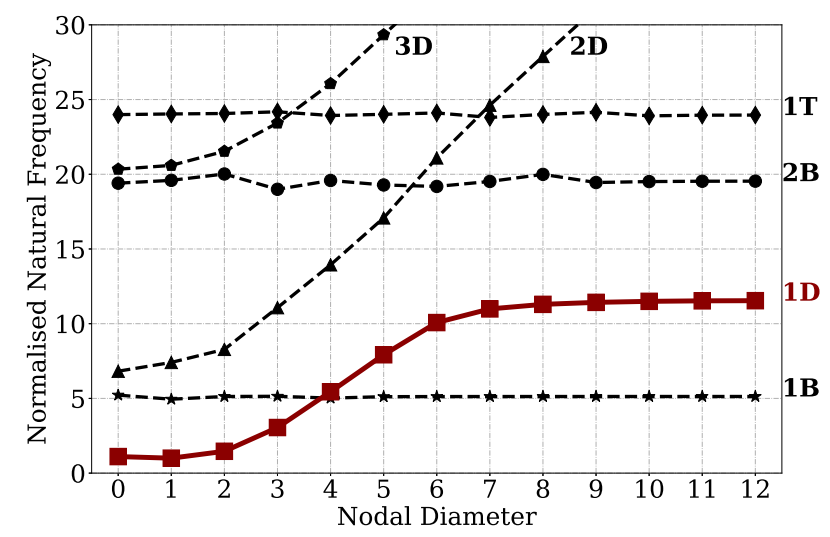

Fig. 5: Natural frequency against nodal diameter of the blisk

\subsection{Campbell diagram}

The Campbell diagram (see Fig.7) shows the change of natural frequencies $\omega_{n}$ against engine rotational speed $\Omega$ and 

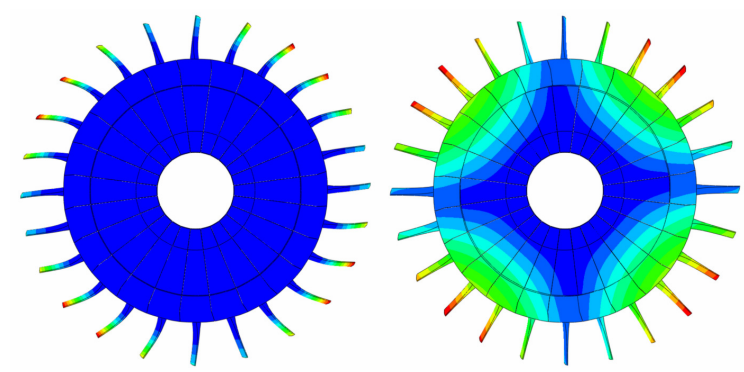

(a) $1^{\text {st }}$ In-plane Bending Mode (1B)

(b) $1^{\text {st }}$ Disk Mode (1D)

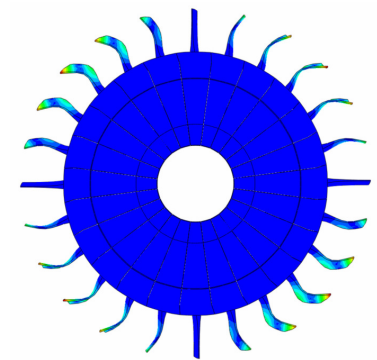

(c) $2^{\text {nd }}$ In-plane Bending Mode (2B)

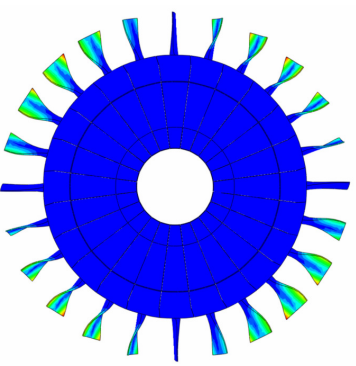

(d) $1^{\text {st }}$ Torsion Mode (1T)

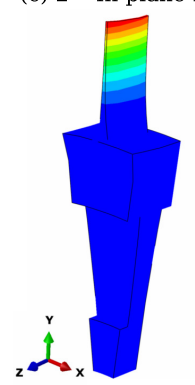

(e) $1 \mathrm{~B}$

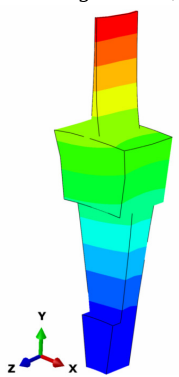

(f) $1 \mathrm{D}$

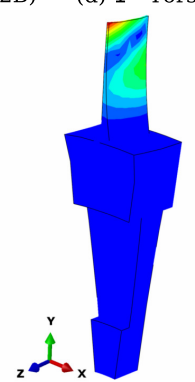

(g) $2 \mathrm{~B}$

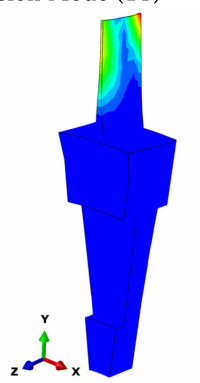

(h) $1 \mathrm{~T}$
Fig. 6: Mode shapes for nodal diameter 2: (a-d) The full blisk; (e-h) A sector

Engine Order (EO) excitation ${ }^{3}$ frequencies. The red lines in Fig.7 represent the change of natural frequencies of the 1D family of modes (all possible NDs). The black lines represent EO excitation which can lead a resonance (marked by blue dot). From the figure, all possible resonance cases for 1D modes are identified. Three resonance cases (marked by blue star, listed in Tab.1) are selected and considered for the subsequent studies.

\subsection{Nonlinear static analysis}

Through the Campbell diagram, the resonances caused by EO excitation are identified and three of the resonance cases are retained for this study. Then, the blisk and ring damper with contact friction under resonance cases are investigated by a static analysis. A surface-to-surface contact model is used and the contact conditions within the contact interface are computed for nonlinear static equilibrium status. A contact pressure distribution and a contact gap dis-

\footnotetext{
${ }^{3}$ Engine order excitation is a common source of excitation in turbomachinery. It is generally caused by the nonuniformity of the flow, due to the nonuniformity of the intake, presence of vanes and rotating bladed disks. The gas steam exerts a non-uniform pressure and flow intensity around the full annulus of the blisk.
}

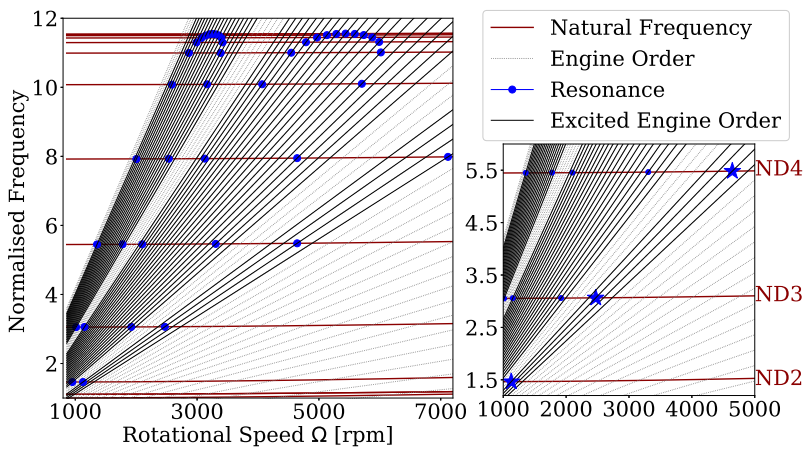

Fig. 7: Campbell diagram of the blisk and a zoom-in for the resonances studied (family of $1^{s t}$ disk mode)

Table 1: Resonance Cases Studied

\begin{tabular}{clll}
\hline Resonance & Case 1 & Case 2 & Case 3 \\
\hline Engine Order & 22 & 21 & 20 \\
Rotational Speed & 1,127 & 2,471 & 4,643 \\
Nodal Diameter & 2 & 3 & 4 \\
Normalised $\omega_{n}$ & 1.46 & 3.06 & 5.48 \\
\hline
\end{tabular}

tribution are used to represent the contact conditions. This static contact conditions are considered as the preloading for the nonlinear vibration analysis.

\section{Nonlinear Vibration Solver}

The FE model of the blisk and ring damper as well as the methodology for pre-processing have been presented in previous sections. The nonlinear vibration solver is introduced in this section. The flow chart of the nonlinear vibration solver is shown in Fig.8. Firstly, reduced model methods applied are introduced. Then, the contact model used in vibration analysis is described. After that, the numerical formulations for the computation of dNNM are well explained.

\subsection{Model reduction}

For the 3D FE model, reduced order modelling is important to reduce the computation costs [27]. Generally, the blisk are assumed to be perfectly tuned structures with cyclic symmetry. The mass and stiffness matrices $\left(\mathbf{M}_{b}, \mathbf{M}_{r}, \mathbf{K}_{b}\right.$, $\mathbf{K}_{r}$ ) of the blisk sector and the sector of ring damper are calculated at the first place. Then, Craig-Bampton method [28] is used to reduce the size of system. The DoFs within contact interface and cyclic boundaries are kept as master DoFs. After that, the cyclic symmetric reduction [25] is applied to reduce left cyclic boundary. The computational time is expected to be reduced to a reasonable level by using such model reduction strategy. The reduced mass and stiffness matrices $\left(\widetilde{\mathbf{M}}_{C B}, \widetilde{\mathbf{K}}_{C B}\right)$ are calculated and used for the subse- 


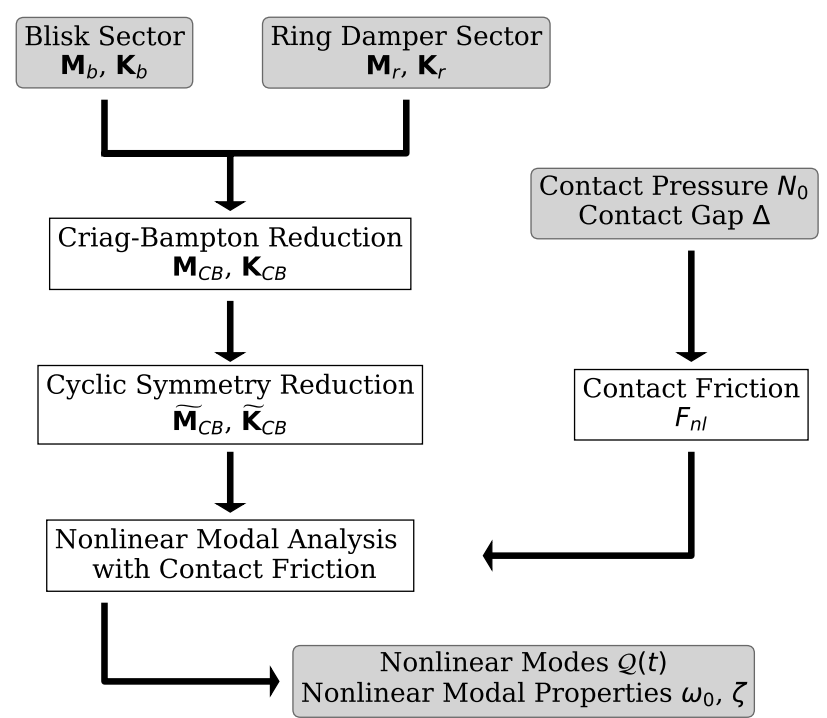

Fig. 8: Flow chart for nonlinear vibration solver

quent studies.

\subsection{Contact model}

To model the contact between the ring damper and the blisk, the combination of two Jenkins elements (in contact plane) and a unilateral spring is used to compute the contact forces between the contact surfaces. This Coulomb-type contact model with varied normal reaction forces was proposed by Yang et al. [6] and is represented in Fig.9. In the normal direction, the unilateral-elastic behaviour with a stiffness $k_{n}$ and a preload $N_{0}$ (or initial gap $\Delta$ for negative $N_{0}$ ) is considered. Whereas, in the tangential direction, elastic dry Coulomb friction with coefficient $\mu$ and stiffness $k_{t 1}$ and $k_{t 2}$ is considered. All the contact parameters are selected based on [4]. In this 3D contact model, a node-to-node contact force is calculated. The preload for each contact node is determined through the nonlinear static analysis. There are 28 nodes within the contact interface (between the underneath of the blisk and upper surface of the ring damper). The overview of contact interface and node number is shown in Fig.3. To understand the contact behaviour within the contact interface, the energy dissipated by each contact node for one vibration period is calculated by simply integrating the tangential friction force with respect to tangential displacement (the area enclosed by hysteresis loop) [29].

\subsection{Nonlinear modal analysis}

The various numerical techniques used for the computation of the dynamic responses of the system with contact model are described in this section. At the first place, the Equation of Motion (EoM) based on the numerical method (EPMC) is described. Then, a well-established HBM for the computation of the dNNMs is presented. After that, the continuation method is described

The EoM for such an autonomous system with contact

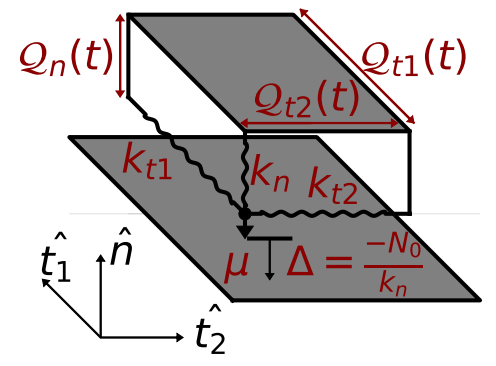

Fig. 9: 3D contact model

friction can be expressed as Eqn.(1), where $\widetilde{\mathbf{M}}_{C B}$ and $\widetilde{\mathbf{K}}_{C B}$, are reduced mass and stiffness matrices respectively. $Q(t)$ is the reduced displacements (including modal coordinates) vector after cyclic symmetric reduction and CB-CMS. $F_{n l}$ corresponds to the friction forces determined with the contact model described above.

$$
\widetilde{\mathbf{M}}_{C B} \ddot{Q}(t)+\widetilde{\mathbf{K}}_{C B} Q(t)+F_{n l}(Q)=0
$$

The properties of the dNNM vary with the level of the energy of the system. To quantify the energy level of the dNNM, a modal amplitude $\alpha$ is introduced into the system. Then, the displacement $Q(t)$ can be presented as $Q(t)=\alpha \times Q_{0}(t)$, where $Q_{0}(t)$ is a mass normalised solution. The system is non-conservative due to the energy lost by contact friction, the free vibration solutions of the system are not periodic. According to EPMC [22], an artificial mass proportional modal damping is introduced into this autonomous system. The artificial damping matrix $\overline{\mathbf{C}}$ is: $\overline{\mathbf{C}}=-2 \omega_{0} \zeta \widetilde{\mathbf{M}}_{C B}$, where $\omega_{0}$ is resonant frequency and $\zeta$ is modal damping ratio. The modal damping ratio $\zeta$ is used to quantify the damping generated by contact friction. Both the resonant frequency $\omega_{0}$ and the modal damping ratio $\zeta$ vary with the energy level of the mode or modal amplitude $\alpha$. Therefore, for a given modal amplitude $\alpha$, the resonant frequency $\omega_{0}$ and the modal damping ratio $\zeta$ are regarded as unknown modal properties and expected to be calculated during the numerical simulation. Hence, the EoM for the computation of dNNM can be written as the equation below. Then, HBM integrated with continuation method is used to solve Eqn.(2) for a range of modal amplitude $\alpha$.

$$
\alpha\left(\widetilde{\mathbf{M}}_{C B} \ddot{Q}_{0}(t)+\overline{\mathbf{C}} \dot{Q}_{0}(t)+\widetilde{\mathbf{K}}_{C B} Q_{0}(t)\right)+F_{n l}\left(\alpha, Q_{0}\right)=0
$$

\subsubsection{Harmonic balance method}

HBM with AFT is used to compute the dNNMs [2,24]. Generally, HBM is also known as Fourier-Galerkin method, through which all the properties in time domain are decomposed by Fourier series truncated to certain order of harmonics $N_{h}$. Hence, the solution of Eqn.(2) can be discretised as Eqn.(3), where $\tilde{Q}_{p}=\tilde{Q}_{p}^{c}-i \tilde{Q}_{p}^{s}$ is the cosine and sine terms associated to the harmonic $p$. 


$$
Q_{0}(t)=\sum_{p=0}^{N_{h}} \operatorname{real}\left\{\tilde{Q}_{p} \cdot\left[\cos \left(p \omega_{0} t\right)+i \sin \left(p \omega_{0} t\right)\right]\right\}
$$

When all properties in time domain are decomposed as Eqn.(3), the EoM in frequency domain is obtained as Eqn.(4), where $\mathbf{A}\left(\omega_{0}, \zeta\right)$ is the dynamic stiffness matrix, $\tilde{Q}$ is the collection of Fourier coefficients of the solutions $\tilde{Q}=\left[\tilde{Q}_{p=0}, \tilde{Q}_{p=1}, \cdots, \tilde{Q}_{p=N_{H}}\right]^{\mathrm{T}} . \quad \tilde{F}_{n l}(\alpha, \tilde{Q})$ is vector of the nonlinear contact friction force given in Fourier basis. It is determined with the AFT procedure [24].

$$
\alpha \mathbf{A}\left(\omega_{0}, \zeta\right) \cdot \tilde{Q}+\tilde{F}_{n l}(\alpha, \tilde{Q})=0
$$

As mentioned above, the $Q_{0}(t)$ is mass normalised solutions. Therefore, an extra constraint for mass normalisation is given as: $\tilde{Q}^{\mathrm{T}} \cdot \widetilde{\mathbf{M}}_{C B} \cdot \tilde{Q}-1=0$. In addition, absolute phase is arbitrary for an autonomous system. Hence, a constraint for phase normalisation is also required as: $\operatorname{imag}\left(\tilde{Q}_{p}^{1}\right)=0$, through which the absolute phase of the first DoF is fixed to zero.

\subsubsection{Continuation method}

For dNNMs, the resonant frequency $\omega_{0}$ and modal damping ratio $\zeta$ are modal amplitude $\alpha$ dependent. Therefore, the continuation method is used to track the evolution of the system behaviour against a specific chasing parameter. In NMA, the dNNMs and other modal properties are expected to be computed for a range of modal amplitude. Readers can refer to [26] for a detailed description and numerical formulation of continuation process. A secant predictor and arc-length corrector are exploited in this work.

\section{Results}

In this section, the three resonance cases listed in Tab.1 are considered and results for the three different geometries of the ring damper presented in Fig.2 through NMA are shown and discussed in the following.

\subsection{Static contact condition}

The static contact conditions from nonlinear static analysis are shown at the first place. The contact pressure and gap distribution at the contact interface are represented in Fig.10 for three ring dampers for the $1^{\text {st }}$ resonance case. The contact interface and contact node is given in Fig.3. The contact pressure distributions are shown in top row and contact gap distribution are shown in bottom row. Generally, the contact pressure is higher in the middle and lower near the boundaries of the sector (along $\mathrm{X}$ axis). This is caused by the higher centrifugal loading in the middle of the blisk due to the existence of blade. By looking at the contact gap, the nodes located on the boundaries of the sector show the larger separation. The contact pressure/gap are used in the contact model as the preloading conditions for NMA.

\subsection{Nonlinear modal analysis}

The numerical methodologies presented in the previous section are applied and for a range of modal amplitude $\alpha$. To quantify the modal amplitude in a physical demonstration, the displacement of the tip of the blade are calculated. The dNNMs and other modal properties, including resonant frequency $\omega_{0}$ and modal damping ratio $\zeta$, are computed for all the three resonance cases and ring dampers. The three resonances cases studied are given in Tab.1 and Fig.2. The modal damping ratio $\zeta$ computed through the NMA is used to quantify the damping generated by the ring damper. The modal properties are shown with respect to the displacement of the tip of the blade. The resonant frequencies $\omega_{0}$ are normalised by the linear natural frequency of the baseline structure, when ring is attached to the blisk and in a static equilibrium status with contact friction. Based on the contact model used, there are three general contact status, namely sticking, sliding and separating. Whereas, the nodes which show both separation and sliding during one vibration period are named as partial-separating-partial-sliding in this work. The evolution of the resonant frequency $\omega_{0}$ and modal damping ratio $\zeta$ for all three geometries are illustrated in Fig.11. The left column shows the change of resonant frequency $\omega_{0}$ against displacement, whereas the right column represents the evolution of the modal damping ratio $\zeta$. At low level of vibration amplitude (small displacement), the contact nodes are either sticking or separating, which depend on the preload applied to each contact node within contact interface. The modal properties in Fig.11 are plotted against the displacement until the maximum modal ratio is achieved. It happens when the whole ring is sliding and a rigid body rotation of the ring occurs. The three rings show the rigid body rotation at different level of displacement leading to the differences in ending displacement.

The variation of the resonant frequency $\omega_{0}$ with respect to the displacement in Fig.11(a, c, e) is typical to a structure with contact friction. Generally, the increase in $\omega_{0}$ in the Fig.11(e) is caused by that those nodes which are separated at static equilibrium and becomes either sticking or sliding. When the nodes start to slide, a softening effect can be observed and the resonant frequency $\omega_{0}$ decreases.

By considering the evolution of the modal damping ratio $\zeta$ for three ring dampers on Fig.11 (b, d, f), one can observe that the damping ability of the three dampers increase when the ND increases. Indeed, for the resonance case $1(N D=2)$, the maximum modal damping ratio is $0.13 \%$. For the resonance case $3(N D=4)$, the maximum modal damping ratio is around $0.21 \%$. At low engine orders, the motion participated by the disk increases with the ND [10]. Therefore, the mode family 1D with ND4 shows higher frictional damping than the case with ND2. Compared among the three ring dampers, the Ring $\mathrm{C}$ shows the maximum modal damping ratio $\zeta$ for all three resonances $(0.13 \%, 0.18 \%$ and $0.21 \%)$.

In Fig.11(e) for Ring B, the resonant frequency $\omega_{0}$ in- 


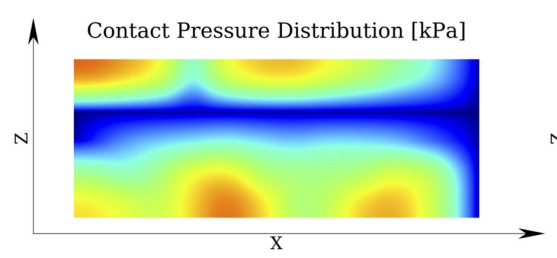

(a) Ring $\mathrm{A}$

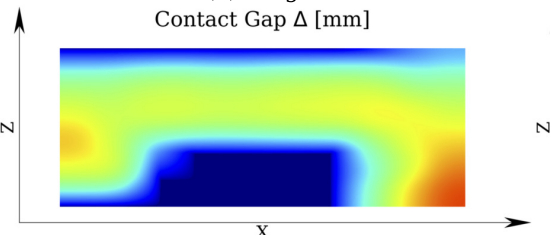

(d) Ring A

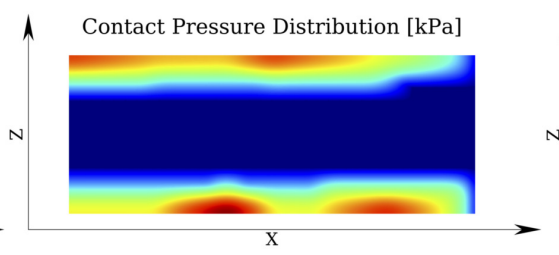

(b) Ring $B$
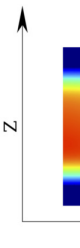

Contact Gap $\Delta[\mathrm{mm}]$

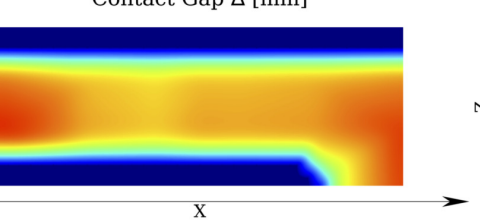

(e) Ring B

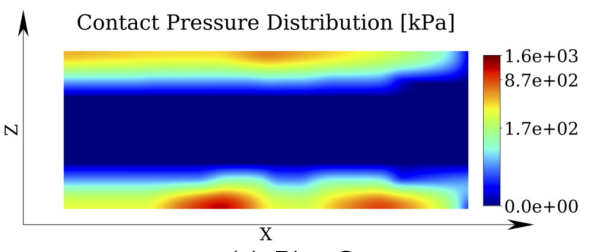

(c) Ring $\mathrm{C}$

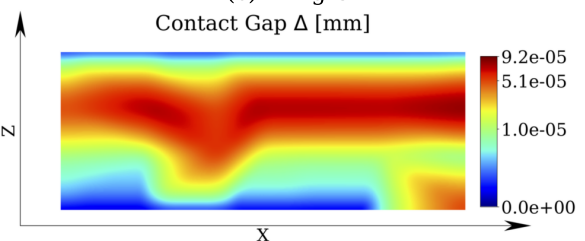

(f) Ring C

Fig. 10: Static contact conditions for the three rings in resonance case 1: (a-c) Contact pressure distribution; (d-f) Contact gap distribution

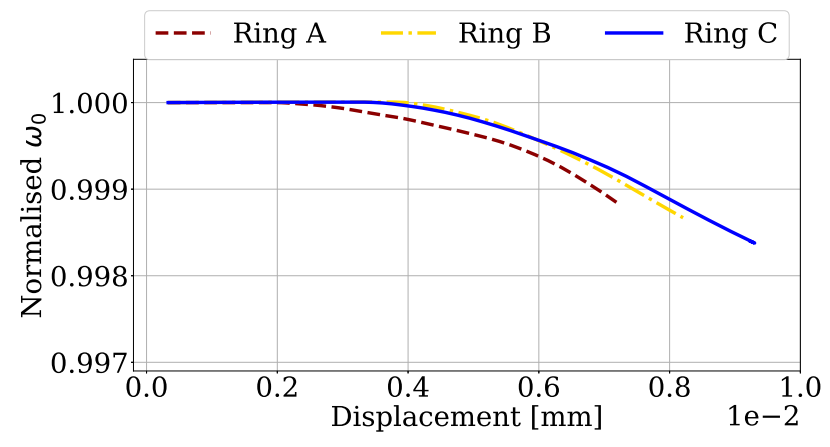

(a) Resonance 1

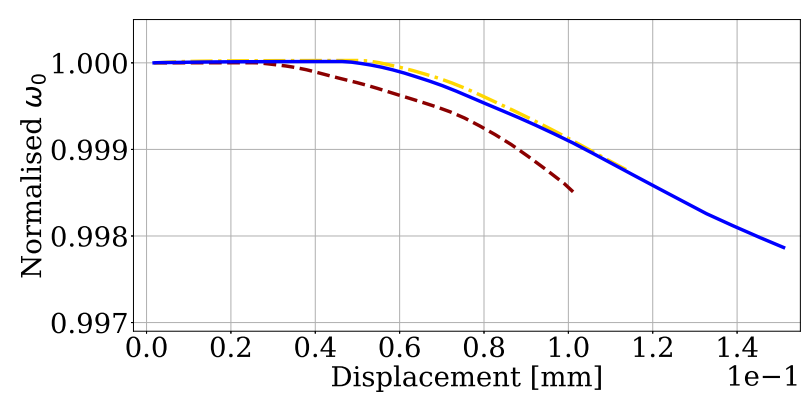

(c) Resonance 2

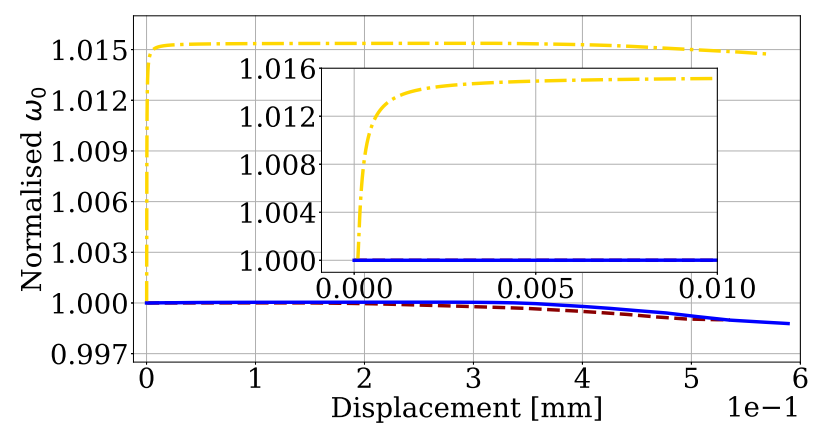

(e) Resonance 3

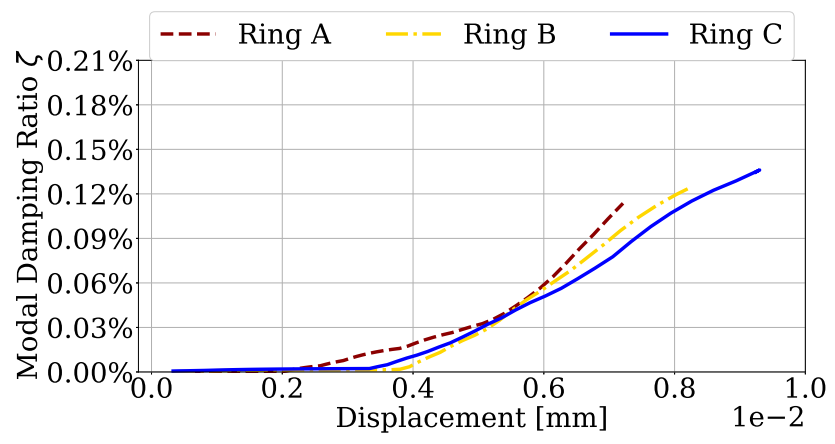

(b) Resonance 1

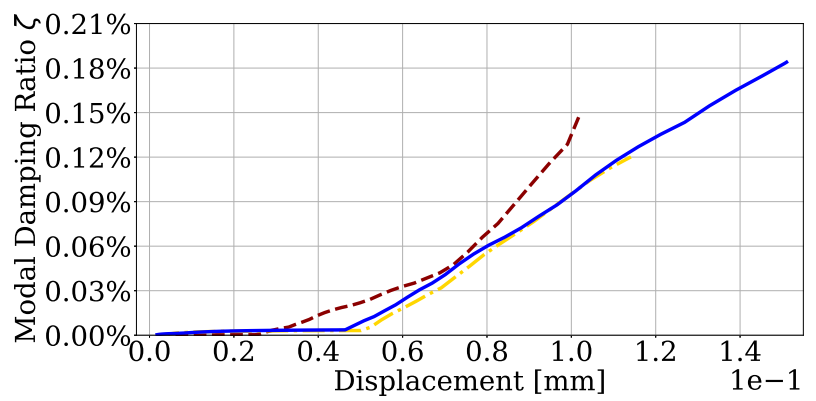

(d) Resonance 2

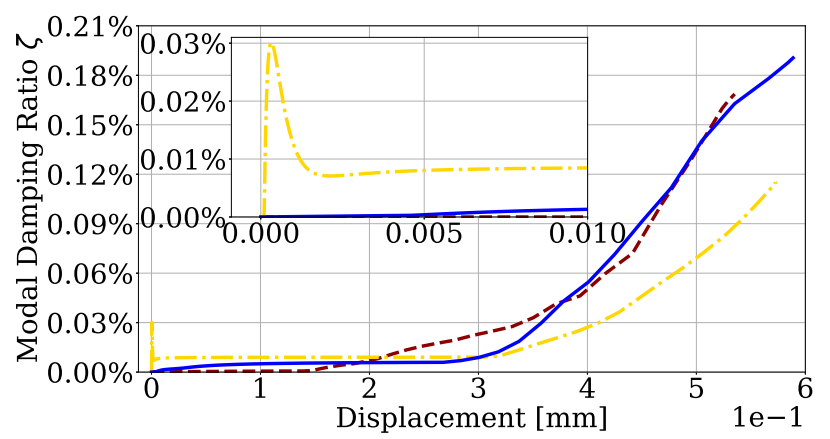

(f) Resonance 3

Fig. 11: Nonlinear modal properties: (a, b) Resonance case 1, (c, d) Resonance case 2, (e, f) Resonance case 3; Left: Normalised resonant frequency; Right: Modal damping ratio 
creases to 1.015 at the level of displacement $0.005 \mathrm{~mm}$. When the system starts to vibrate at low level of amplitude, those separated nodes with small initial gap become incontact and a larger stiffness is achieved leading to a higher resonant frequency. As for modal damping ratio $\zeta$, a small peak is observed in low level of amplitude and the zoom-in of the peak is also shown in the Fig.11(f). The modal damping ratio $\zeta$ increases to $0.03 \%$ and gradually reduce to value around $0.008 \%$. The separated nodes with small gap start to slide at low amplitude and the frictional damping is generated. In this case, the nodes are partial-separating-partialsliding within one period of vibration. The modal damping ratio for these nodes increases with the proportion of the sliding status. The gradual decrease of the modal damping ratio is caused by increased proportion of the separation in partial-separating-partial-sliding. The similar phenomenon was found and described in [30].

\subsection{Dynamic contact condition}

The evolution of the modal damping ratio $\zeta$ and resonant frequency $\omega_{0}$ with respect to the displacement have been described above. To further investigate the contact phenomenon, the evolution of contact status and energy dissipated for each contact node are considered for the three ring dampers under resonance case 3 as shown in Fig.12. The left column in the figure (a, c, e) demonstrates the evolution of the contact status for all contact nodes. In Fig.12 (a, c, e), red circles represent the separation; blue squares are partial-separating-partial-sliding; black triangles are sliding status; the sticking status are left as no markers. The right column (b, d, f) shows the energy dissipated for each contact node, the bandwidth and colour represent the magnitude of the energy dissipated. The contact node map is given in Fig.3.

In Fig. 12 (b, d, f), it is obvious that the Ring $\mathrm{C}$ shows largest amount of energy dissipated, which corresponds to the results in Fig.11(f). By considering both contact status and energy dissipation, the higher level of energy dissipation can be observed in nodes which are sliding (black triangles, i.e. node 1, 4, 8 for Ring C). The energy dissipated is zero for the sticking nodes and separated nodes (red circles). For the nodes which are partial-separating-partial-sliding (blue squares), the energy dissipated is in a relatively low level compared to the sliding nodes. Taking the node 6 (partialseparating-partial-sliding node) for Ring $\mathrm{C}$ as an example, the energy dissipated for node 6 at maximum displacement is $2 e-3 \mathrm{~J}$, which is much lower than $5.7 e-2 \mathrm{~J}$ the value of node 8 (sliding node).

The evolution of resonant frequency and modal damping ratio for Ring $\mathrm{B}$ under resonance case 3 are discussed in previous section. The evolution of the contact status for Ring B are shown in Fig.12(c). Compared to other two rings, these separated nodes in Ring B (i.e. node 2, 3) become partial-separating-partial-sliding at lower amplitude. Once they transit from separation to partial-separatingpartial-sliding, the system becomes stiffer leading to an increase in the resonant frequency. The energy is dissipated

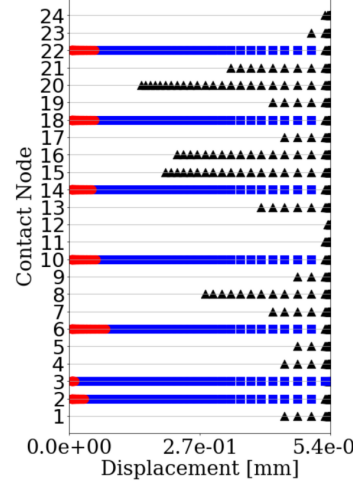

(a) Ring A

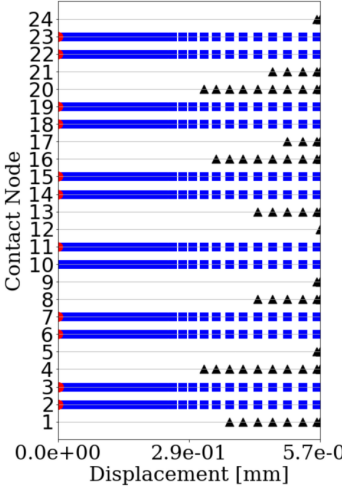

(c) Ring B

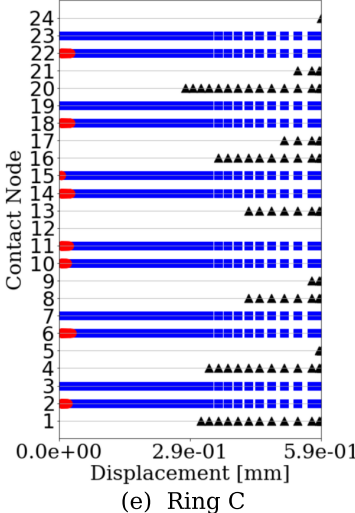

(e) Ring C

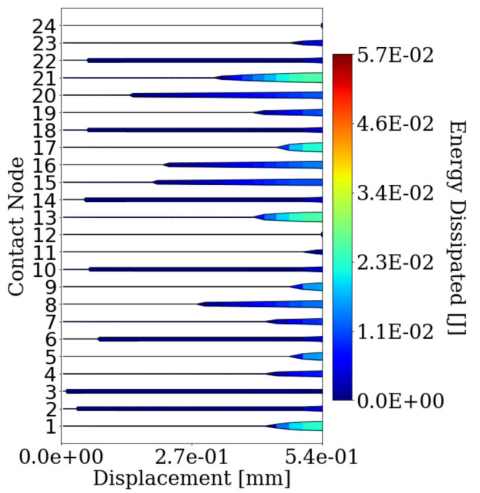

(b) Ring A

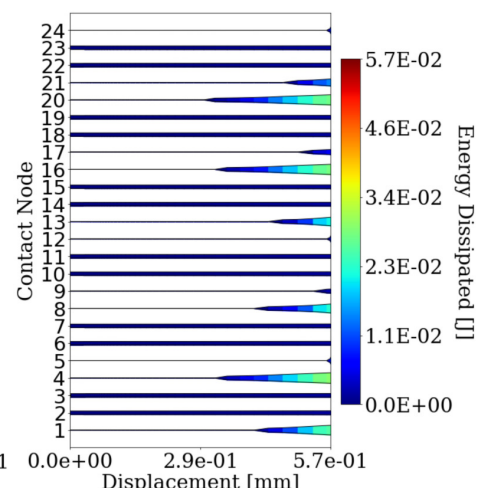

(d) Ring B

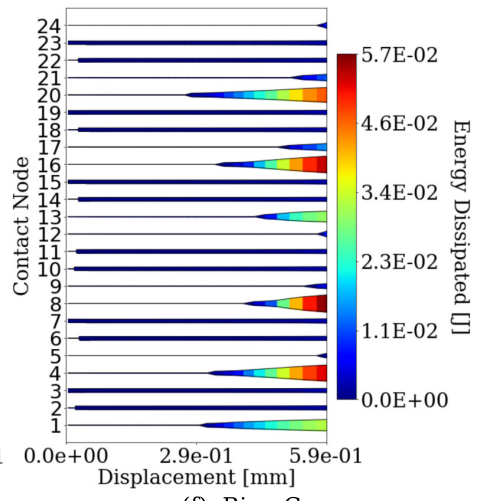

(f) Ring $\mathrm{C}$
Fig. 12: Contact conditions for three rings, resonance case 3: Left: Contact status (separation $\bigcirc$, partial-separationpartial-sliding $\square$, sliding $\mathbf{\Lambda}$; Right: Energy dissipation

by those nodes, which are partial-separating-partial-sliding. Therefore, a positive modal damping ratio is obtained.

\section{Conclusion}

A three-dimensional finite element model is used in this work to represent the integrally blisk and ring damper. Three different ring dampers are studied through nonlinear modal analysis and damping abilities are assessed and compared using the modal damping ratio. The maximum modal damping ratio can be efficiently identified through nonlinear modal analysis when the full ring has a rigid body rotation. The general damping achieved by ring damper is around $0.1 \%$ to $0.2 \%$. The ring with curved contact surface (Ring C) has the 
best damping performance among three ring dampers studied in this work. The damping ability varies with mode and nodal diameter. The contact pressure and gap within the contact interface also have significant impact on the damping performance. Separations within contact interface are expected to be avoided to achieve a higher damping performance. A topological optimisation for the cross section of the ring damper is considered as the future work.

\section{Acknowledgements}

The first author is grateful to China Scholarship Council (File NO. 201708060239) for providing the financial support for this project.

\section{References}

[1] Yuan, J., Scarpa, F., Allegri, G., Titurus, B., Patsias, S., and Rajasekaran, R., 2017. "Efficient computational techniques for mistuning analysis of bladed discs: A review". Mechanical Systems and Signal Processing, 87, pp. $71-90$.

[2] Petrov, E. P., and Ewins, D. J., 2003. “Analytical formulation of friction interface elements for analysis of nonlinear multi-harmonic vibrations of bladed disks". ASME J Turbomach, 125(2), pp. 364 - 371.

[3] Krack, M., Salles, L., and Thouverez, F., 2016. "Vibration prediction of bladed disks coupled by friction joints". Archives of Computational Methods in Engineering, 24, pp. $589-636$.

[4] Pesaresi, L., Salles, L., Jones, A., Green, J. S., and Schwingshackl, C. W., 2017. "Modelling the nonlinear behaviour of an underplatform damper test rig for turbine applications". Mechanical Systems and Signal Processing, 85, pp. $662-679$.

[5] Pesaresi, L., Armand, J., Schwingshackl, C., Salles, L., and Wong, C., 2018. "An advanced underplatform damper modelling approach based on a microslip contact model". Journal of Sound and Vibration, 436, pp. $327-340$.

[6] Yang, B. D., and Menq, C. H., 1998. "Characterization of $3 \mathrm{~d}$ contact kinematics and prediction of resonant response of structures having $3 \mathrm{~d}$ frictional constraint". Journal of Sound and Vibration, 217(5), pp. 909 - 925.

[7] Niemotka, M. A., and Ziegert, J. C., 1995. "Optimal design of split ring dampers for gas turbine engines". ASME J Eng Gas Turb Power, 117(3), pp. 569 - 575.

[8] Zucca, S., Firrone, C. M., and Facchini, M., 2010. "A method for the design of ring dampers for gears in aeronautical applications". ASME. Paper No.IMECE2010 38788.

[9] Wang, Y., Ye, H., Jiang, X., and Tian, A., 2018. "A prediction method for the damping effect of ring dampers applied to thin-walled gears based on energy method". Symmetry, 10(12), p. 677.

[10] Laxalde, D., Thouverez, F., Sinou, J.-J., and Lombard, J.-P., 2007. "Qualitative analysis of forced response of blisks with friction ring dampers". European Journal of Mechanics - A/Solids, 26(4), pp. 676-687.

[11] Sun, Y., Yuan, J., Pesaresi, L., and Salles, L., 2018. "Nonlinear vibrational analysis for integrally bladed disk using frictional ring damper". Journal of Physics: Conference Series, 1106, p. 012026.

[12] Laxalde, D., Thouverez, F., and Lombard, J.-P., 2010. "Forced response analysis of integrally bladed disks with friction ring dampers". ASME J Vib Acoust, 132(1), p. 011013.

[13] Baek, S., and Epureanu, B., 2017. "Reduced-order modeling of bladed disks with friction ring dampers". ASME J Vib Acoust, 139(6), p. 061011.

[14] Lupini, A., Mitra, M., and Epureanu, B. I., 2019. "Application of tuned vibration absorber concept to blisk ring dampers: A nonlinear study". ASME J Eng Gas Turb Power, 141(10), p. 101016.

[15] Wu, Y., Li, L., Fan, Y., Zucca, S., Gastaldi, C., and Ma, H., 2020. "Design of dry friction and piezoelectric hybrid ring dampers for integrally bladed disks based on complex nonlinear modes". Computers and Structures, 233, p. 106237.

[16] Laxalde, D., Gibert, C., and Thouverez, F., 2008. "Experimental and numerical investigations of friction rings damping of blisks". ASME. Paper No.GT2008 50862.

[17] Tang, W., and Epureanu, B. I., 2019. "Geometric optimization of dry friction ring dampers". International Journal of Non-Linear Mechanics, 109, pp. 40 - 49.

[18] Rosenberg, R. M., 1960. "Normal modes of nonlinear dual-mode systems". ASME J Appl Mech, 27(2), pp. $263-268$.

[19] Shaw, S. W., and Pierre, C., 1991. "Non-linear normal modes and invariant manifolds". Journal of Sound and Vibration, 150(1), pp. $170-173$.

[20] Sarrouy, E., 2019. "Phase driven modal synthesis for forced response evaluation". In 7 th International Conference on Nonlinear Vibrations, Localization and Energy Transfer.

[21] Laxalde, D., Salles, L., Blanc, L., and Thouverez, F., 2008. "Non-linear modal analysis for bladed disks with friction contact interfaces". ASME. Paper No. GT2008 $-50860$

[22] Krack, M., 2015. "Nonlinear modal analysis of nonconservative systems: Extension of the periodic motion concept". Computers and Structures, 154, pp. $59-71$.

[23] Krylov, N., and Bogoliubov, N., 1943. Introduction to Non-linear Mechanics. Annals of mathematics studies. no.11. Princeton University Press.

[24] Cameron, T. M., and Griffin, J. H., 1989. "An alternating frequency/time domain method for calculating the steady-state response of nonlinear dynamic systems". ASME J Appl Mech, 56(1), pp. 149 - 154.

[25] Petrov, E. P., 2004. "A method for use of cyclic symmetry properties in analysis of nonlinear multiharmonic vibrations of bladed disks". ASME J Turbomach, 126(1), pp. 175 - 183.

[26] Sarrouy, E., and Sinou, J.-J., 2011. "Non-linear peri- 
odic and quasi-periodic vibrations in mechanical systems - on the use of the harmonic balance methods". In Advances in Vibration Analysis Research, F. Ebrahimi, ed. InTech, Rijeka, ch. 21.

[27] Yuan, J., El-Haddad, F., Salles, L., and Wong, C., 2019. "Numerical assessment of reduced order modeling techniques for dynamic analysis of jointed structures with contact nonlinearities". ASME. Paper No. GT2018 - 75303.

[28] Craig, JR., R. R., and Bampton, M. C. C., 1968. "Coupling of substructures for dynamic analyses". AIAA Journal, 6(7), pp. 1313 - 1319.

[29] Firrone, C., and Zucca, S., 2011. "Modelling friction contacts in structural dynamics and its application to turbine bladed disks". Numerical Analysis: Theory and Application, 14, pp. $301-334$.

[30] Sun, Y., Yuan, J., Pesaresi, L., Denimal, E., and Salles, L., 2020. "Parametric study and uncertainty quantification of the nonlinear modal properties of frictional dampers". ASME J Vib Acoust, 142(5), p. 051102. 